\title{
Influence of Stationary Crossflow Modulation on Secondary Instability
}

\author{
Meelan M. Choudhari ${ }_{2}^{1} \mathrm{Fei} \mathrm{Li}_{2}{ }^{2}$ and Pedro Paredes ${ }^{3}$ \\ NASA Langley Research Center, Hampton, VA, 23681
}

\begin{abstract}
A likely scenario for swept wing transition on subsonic aircraft with natural laminar flow involves the breakdown of stationary crossflow vortices via high frequency secondary instability. A majority of the prior research on this secondary instability has focused on crossflow vortices with a single dominant spanwise wavelength. This paper investigates the effects of the spanwise modulation of stationary crossflow vortices at a specified wavelength by a subharmonic stationary mode. Secondary instability of the modulated crossflow pattern is studied using planar, partial-differential-equation based eigenvalue analysis. Computations reveal that weak modulation by the first subharmonic of the input stationary mode leads to mode splitting that is particularly obvious for Y-type secondary modes that are driven by the wall-normal shear of the basic state. Thus, for each Y mode corresponding to the fundamental wavelength of results in unmodulated train of crossflow vortices, the modulated flow supports a pair of secondary modes with somewhat different amplification rates. The mode splitting phenomenon suggests that a more complex stationary modulation such as that induced by natural surface roughness would yield a considerably richer spectrum of secondary instability modes. Even modest levels of subharmonic modulation are shown to have a strong effect on the overall amplification of secondary disturbances, particularly the Z-modes driven by the spanwise shear of the basic state. Preliminary computations related to the nonlinear breakdown of these secondary disturbances provide interesting insights into the process of crossflow transition in the presence of the first subharmonic of the dominant stationary vortex.
\end{abstract}

\section{Nomenclature}

| $A_{\text {init }}=$ initial amplitude of crossflow instability mode or secondary instability mode, measured in terms of peak chordwise velocity perturbation and normalized with respect to freestream velocity

$C=$ wing chord length normal to the leading edge

$f \quad=$ frequency of instability oscillations $(\mathrm{Hz})$

$M \quad=$ freestream Mach number

$N=\mathrm{N}$-factor (i.e., logarithmic amplification ratio) of linear crossflow instability or secondary instability

$\mid(m, n)=$ temporal and spatial Fourier mode number pair

$(u, v, w)=$ Cartesian velocity components aligned with $(x, y, z)$ axes

$X \quad=$ chordwise coordinate in direction perpendicular to leading edge

$\mid \underline{Y}=$ wall-normal coordinate

$Z=$ spanwise coordinate, i.e., the coordinate parallel to airfoil leading edge

$\lambda=$ spanwise wavelength of crossflow instability

Abbreviations

NPSE $=$ nonlinear parabolized stability equations

DNS $\quad=$ direct numerical simulations

${ }_{1}^{1}$ Aerospace Technologist, MS 128, Meelan.M.Choudhari@nasa.gov, Associate Fellow, AIAA.

2 Aerospace Technologist. MS 128, Fei.Li@nasa.gov.

${ }^{3}$ NASA NPP Fellow, MS 128, Pedro.Paredesgonzalez@nasa.gov, AIAA Member. 


\section{Introduction}

$\mathrm{T}$ HE economic and environmental benefits of laminar flow technology via reduced fuel burn of subsonic and supersonic aircraft cannot be realized without reducing the uncertainty in drag prediction in general and transition prediction in particular. Transition research under NASA's Transformational Tools and Technologies Project seeks to develop a validated set of variable fidelity prediction tools with known strengths and limitations, so as to enable sufficiently accurate transition prediction and practical transition control for future vehicle concepts. The purpose of the present research is to investigate transition mechanisms involving a spanwise modulated train of stationary crossflow vortices of a specified dominant wavelength, which have received little attention in comparison with stationary vortices with a single spanwise wavelength.

In general, a swept wing boundary layer can be susceptible to various types of primary instabilities such as attachment line instability ${ }^{1}$, stationary and traveling crossflow modes, and Tollmien-Schlichting (TS) waves. Regardless of which mode dominates the primary amplification stage, the other, subdominant modes may play a role during the nonlinear stage, and hence, influence the onset of transition. A likely scenario during the flight of sweptwing aircraft involves the breakdown of stationary crossflow vortices via high frequency secondary instability. ${ }^{1-6}$ Transition prediction based on the linear amplification of secondary instabilities accounts for the nonlinear evolution of crossflow vortices and, unlike the predictions based on vortex amplitudes themselves, provides an adequately sensitive measure to predict the onset of transition. Application of this technique to the flight experiment by Carpenter et al. ${ }^{7}$ is described by Li et al. ${ }^{8}$ Nonlinear development of the secondary instability modes of stationary vortices in the boundary layer flow over a transport relevant infinite-swept wing configuration has also been addressed using nonlinear parabilized stability equations (NPSE) ${ }^{9}$ and direct numerical simulations (DNS).$^{10,11}$

Even if the boundary layer flow over a swept airfoil is homogeneous along the spanwise direction, the surface finish characteristics may involve noticeable inhomogeneities along the span. As a result, the naturally excited crossflow vortices often involve a spanwise modulation of the dominant crossflow wavelength such that the adjacent vortices are not identical to each other, and display pronounced variations from one vortex to the next. To model the effects of this spanwise modulation in the simplest possible context, we employ a flow setting that is analogous to the "multiple-mode crossflow wave" configuration of Reibert and Saric ${ }^{12}$ wherein crossflow disturbances at a subharmonic spacing of the most amplified stationary mode were seeded along with the crossflow disturbance at the fundamental wavelength. This paper models a similar scenario for a realistic, transport relevant swept airfoil configuration. Whereas Reibert and Saric used a roughness array spacing of three times the wavelength of the dominant crossflow mode, we find it sufficient to seed the first subharmonic to induce the necessary modulation of the stationary crossflow modes over the flow configuration of interest.

A brief description of the infinite swept wing configuration of interest and the analysis tools employed in the present work is given in Section II. The combined nonlinear evolution of the fundamental and subharmonic crossflow modes is addressed in Section III by using NPSE. While keeping the initial amplitude of the dominant stationary mode fixed, the strength of the spanwise modulation is varied by introducing a subharmonic mode with varying initial amplitude and/or phase with respect to the dominant crossflow wavelength (Section III-A). The secondary instability characteristics of the resulting patterns of crossflow vortices are studied by solving a planar, partial differential equation based spatial eigenvalue problem that accounts for the disparity between the vortex orientation and the chordwise direction. ${ }^{6}$ The mode shapes of the predicted secondary modes for various levels of spanwise modulations are scrutinized in Sections III-C through III-E, and are also compared with the secondary instabilities in the simpler case of unmodulated crossflow vortices (Section III-B). Preliminary results pertaining to the nonlinear evolution of these secondary instability modes are presented in Section IV. A related accompanying paper $^{13}$ extends the prior studies of the same airfoil configuration by investigating the effects of subharmonic wavelength modes of the high frequency secondary instability, and the combined evolution of multiple types of secondary instability modes.

\section{Flow Configuration and Analysis Codes}

The flow configuration of interest in this paper is identical to the laminar flow airfoil TAMU-003T-75(v.90) that was previously used by Duan et al. ${ }^{10}$ and Choudhari et al. ${ }^{11}$ to explore the breakdown characteristics of isolated Yand Z-modes, respectively, of the secondary instability of stationary crossflow modes with a specified wavelength. The design process for this laminar flow airfoil was described by Belisle et al. ${ }^{14}$ The 9.3 percent thick, 30-degree swept airfoil is designed to achieve natural laminar flow over 60 and 50 percent of the suction and pressure surfaces, respectively, at the design condition of zero degree angle of attack, Mach number of $\mathrm{M}=0.75$, and chord Reynolds number of approximately 17 million based on freestream speed, a streamwise chord length of $3.658 \mathrm{~m} \mathrm{(12} \mathrm{ft),}$ freestream temperature of $216.65 \mathrm{~K}$, and density of $0.302 \mathrm{~kg} / \mathrm{m}^{3}$. The latter conditions correspond to a flight altitude 
of $12,192 \mathrm{~m}(40,000 \mathrm{ft})$. Design constraints for the wing included a lift coefficient that is typical of subsonic transports and a wing thickness distribution that is suitable for a mid-size business jet. At the design angle of attack, both Tollmien-Schlichting and crossflow instabilities are sufficiently weak that natural laminar flow should be achievable over a significant portion of the airfoil surface without any external means of boundary layer control. In this paper, we focus on the off design condition corresponding to an angle of incidence equal to -1 degree, i.e., the same condition as that used by Li et al. ${ }^{9}$ in their PSE and LSIT analysis. At this off-design condition, a stronger crossflow instability exists on the suction side, ${ }^{9,14}$ which provides the basis for potentially implementing DRE-based laminar flow technology at the higher Reynolds numbers of interest.

For the work described in this paper, the mean boundary-layer flow over the suction surface of the airfoil was taken from the boundary layer solution obtained by $\mathrm{Li}$ et al. ${ }^{9}$ It was computed using the boundary layer solver BLSTA, ${ }^{15}$ designed specifically for generating accurate boundary layer solutions that are required for boundary layer stability analysis of swept and tapered wings. The inviscid surface-pressure distribution was derived from an Euler solution under free flight conditions. ${ }^{14}$ The number of wall-normal points used for boundary layer computations was well in excess of that used by $\mathrm{Wie}^{15}$ for validating the BLSTA code. Sensitivity to streamwise resolution was also assessed by Li et al. ${ }^{9}$ by using $115,229,457$ and 913 points over the chord length of interest (with proportionally greater number of points near the leading edge), and the solution was converged with 115 streamwise points. Similar to the preceding work, ${ }^{9}$ the computations presented in this paper are based on 229 points over the chord length. The boundary layer solution computed in this manner is suitably interpolated using cubic splines when necessary.

The growth of high-frequency secondary instability modes supported by a uniform pattern of finite amplitude stationary crossflow vortices of fixed spanwise wavelength was analyzed by Li et al. ${ }^{9}$ by using the fully spatial methodology described in the earlier papers. ${ }^{6}$ The main difference between the primary and secondary stability analyses is that the basic state for the secondary modes (i.e., the mean boundary layer flow modified by the primary crossflow mode) varies in both surface-normal and spanwise directions; and hence, the instability characteristics of the secondary modes must be analyzed using a planar, partial differential equation based eigenvalue problem, rather than the ordinary differential equation based eigenvalue problem of the classical analysis. In ongoing work, nonparallel effects on the secondary mode evolution are being evaluated using plane marching PSE ${ }^{16}$ and the results of that analysis will be presented in a future paper. The selection of the grid and other aspects of the numerical computation of secondary instabilities was based on extensive experience with a similar class of flows, and spot checks were made to ensure that the amplification rates predicted via baseline grids were in agreement with those based on grids that were twice as large in either direction. Typically, 121 points in the wall-normal direction and 65 points in the spanwise direction were found to be sufficient for the 2D, planar eigenvalue analysis involving subharmonic modulation of the stationary state.

Linear and nonlinear development of the primary instability (i.e., stationary crossflow vortices) is computed using linear/nonlinear parabolized stability equations (LPSE and NPSE, respectively) as implemented in the Langley Stability and Transition Analysis Codes (LASTRAC). ${ }^{17}$ For nonlinear PSE marching, 281 points are used in the wall-normal direction, which is more than sufficient. The streamwise marching step-size was appropriately adjusted for each computation as needed to capture the corresponding modal evolution. We denote the ranges of Fourier modes in time and in the spanwise direction by $(-m, m)$ and $(-n, n)$, respectively. A total of 48 spanwise Fourier harmonics $(n=96)$ is used to compute the nonlinear development of a primary crossflow mode of specified spanwise wavelength, $\lambda$, and the truncation error is estimated a posteriori to be at least nine orders of magnitude smaller than the most energetic fundamental mode. Linear and nonlinear development of the secondary instabilities of crossflow vortices and the ensuing laminar breakdown process is predicted by using six to twelve temporal harmonics $(n=6$ to 12), with additional harmonics introduced as required during the course of the chordwise marching computation.

\section{Secondary Instability of Modulated Crossflow Patterns}

As described in a previous paper by Li et al., ${ }^{9}$ the swept wing boundary layer of interest supports stationary crossflow instabilities over a broad spectrum of spanwise wavelengths, ranging from approximately $1 \mathrm{~mm}$ to more than $30 \mathrm{~mm}$. The most amplified stationary crossflow mode corresponds to $\lambda=8 \mathrm{~mm}$, which achieves a peak linear $\mathrm{N}$-factor of more than 12. Its first subharmonic at $\lambda=16 \mathrm{~mm}$, which is used in this paper to provide the necessary spanwise modulation for the $8 \mathrm{~mm}$ mode, also has a strong linear growth potential, reaching a peak $\mathrm{N}$-factor of approximately nine.

\section{A. Nonlinear Evolution of $\lambda=8 \mathrm{~mm}$ mode modulated by $\lambda=16 \mathrm{~mm}$}

Figure 1 shows the NPSE predictions for the combined evolution of stationary crossflow modes for specified initial amplitudes of the $\lambda=8 \mathrm{~mm}$ and $\lambda=16 \mathrm{~mm}$ modes. In all cases, the initial amplitude of the fundamental 
stationary crossflow mode $(\lambda=8 \mathrm{~mm})$ is held fixed while the amplitude of the subharmonic $\lambda=16 \mathrm{~mm}$ mode is varied in order to assess the effects of spanwise modulation. Figure 1(a) shows the modal evolution as a function of the (normalized) chordwise coordinate $X / C$ for $\lambda=8 \mathrm{~mm}$ and $\lambda=16 \mathrm{~mm}$, along with the corresponding variation in the amplitude of the mean flow correction, MFC, which provides an approximate measure of the level of nonlinear effects on the stationary crossflow evolution. The baseline case with zero modulation is denoted as case B in Fig. 1. Three additional cases corresponding to increasing modulation amplitudes are included in the plot, which are denoted as L (low), M (mid), and $\mathrm{H}$ (high) level of modulation, respectively. These cases are defined in terms of the amplitudes of the fundamental and subharmonic modes at $X / C=0.20$, where these modes reach an amplitude of $\underline{\mathrm{O}(1 \%), \text { which is deemed amenable to measurement in an experiment. In all four cases, the amplitude of the } \lambda=8}$ mm mode at $X / C=0.20$ is 0.0255 , whereas the modulation amplitude varies through 0 (Case B), 0.00088 (Case $\mathrm{L}$ ), 0.00176 (Case M) and 0.00325 (Case H), respectively. Here, each modal amplitude represents the maximum perturbation in chordwise velocity component, normalized by the freestream speed $U_{\infty}$. In all three cases, the phase difference between the fundamental and subharmonic modes at the inflow location of $X / C=0.0212$ is set to zero. For the highest level of modulation (Case $\mathrm{H})$, the amplitude of the $\lambda=16 \mathrm{~mm}$ mode is sufficiently high for this mode to dominate over the $\lambda=8 \mathrm{~mm}$ mode; and hence, this case provides an approximate upper bound on the level of modulation to be used in the present study.

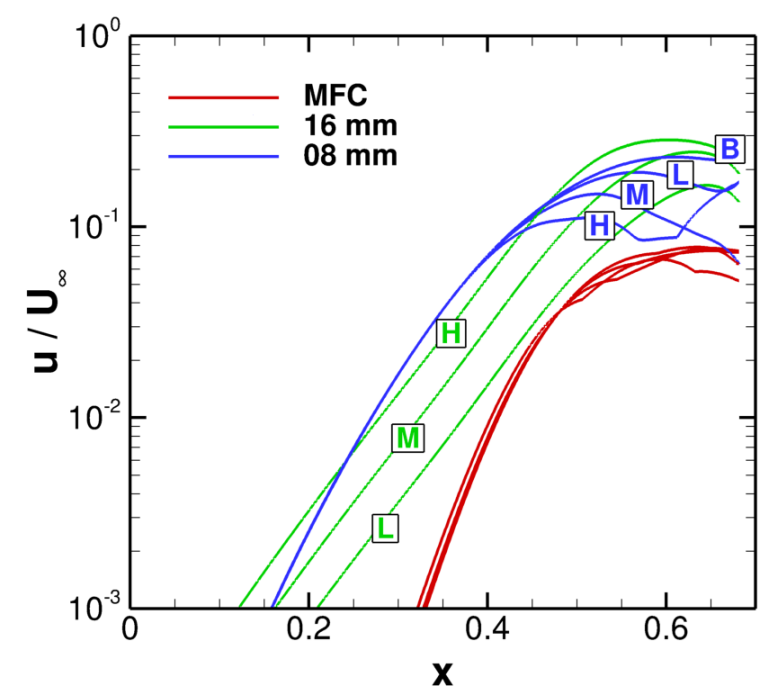

(a) Effect of modulation strength (characterized by the initial amplitude of the $\lambda=16 \mathrm{~mm}$ mode) for zero initial phase difference. MFC denotes mean flow correction.

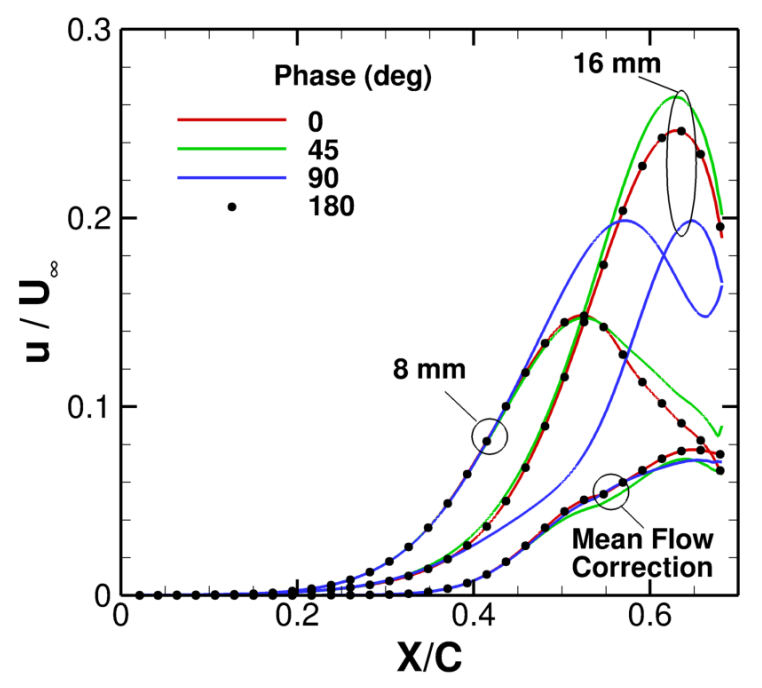

(b) Effect of relative initial phase between the $\lambda=8$ $\mathrm{mm}$ and $\lambda=16 \mathrm{~mm}$ modes for case $M$.

Figure 1. Nonlinear evolution of $\lambda=8 \mathrm{~mm}$ stationary mode with modulation by $\lambda=16 \mathrm{~mm}$ mode.

The modal evolutions plotted in Fig. 1(b) correspond to the case where the amplitudes of both fundamental $(\lambda=$ $8 \mathrm{~mm})$ and subharmonic $(\lambda=16 \mathrm{~mm})$ modes have been held fixed, but the relative phase of the subharmonic mode at the inflow station is varied from 0 degrees to 180 degrees. The figure shows that (among the phase values considered herein) a relative phase of 90 degrees leads to the highest growth of the fundamental mode and the lowest peak amplitude of the subharmonic mode. On the other hand, a relative phase angle of 45 degrees is seen to maximize the spanwise modulation associated with the $\lambda=16 \mathrm{~mm}$ mode. Both modes evolve in an identical manner for relative phase angles of 0 degrees and 180 degrees, which is to be expected because the $\lambda=8 \mathrm{~mm}$ mode corresponds to first harmonic of the $\lambda=16 \mathrm{~mm}$ mode; and hence, the initial condition for a phase difference of 0 degrees can be recovered via $8 \mathrm{~mm}$ spanwise translation of the initial condition corresponding to a phase difference of 180 degrees. The results in Fig. 1(b) show that the initial phase difference can have a marked effect on the downstream evolution of the fundamental mode and the modulation. However, the effect of phase differences on the secondary instability of the resulting vortex structures is deferred to a future investigation and the remaining paper focuses entirely on the cases described in Fig. 1(a). 
B. Secondary Instability for Zero Modulation Case (Case B)

Figures 2(a) through 2(d) show the crossplane contours of normalized chordwise velocity at selected stations ranging from $X / C=0.40$ to $X / C=0.65$. While the velocity contours at $X / C=0.40$ indicate a relatively benign vortex structure, the strongly overturned (i.e., rolled up) contours at the remaining three locations indicate the potential for a strong, high-frequency secondary instability along the outer shear layer that bounds the vortex structure. The results of secondary instability analysis at $X / C=0.60$ are shown in Figs. 3(a) and (b). Illustrative mode shapes depicting the cross-sectional distribution of the chordwise velocity fluctuation associated with the different secondary mode disturbances are shown in Figs. 4(a) through 4(e).

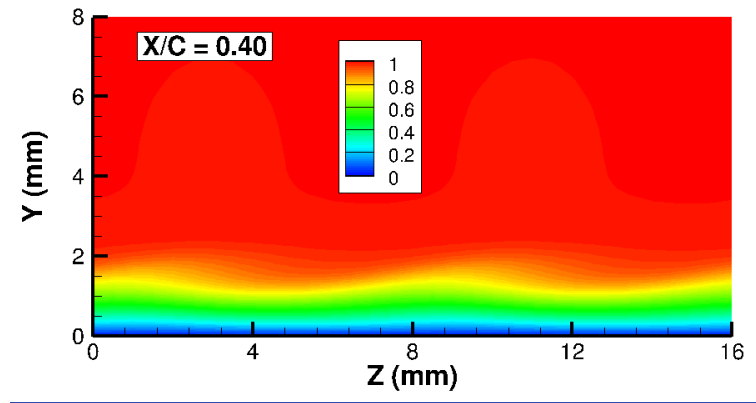

(a) $\mathrm{X} / \mathrm{C}=\mathbf{0 . 4}$

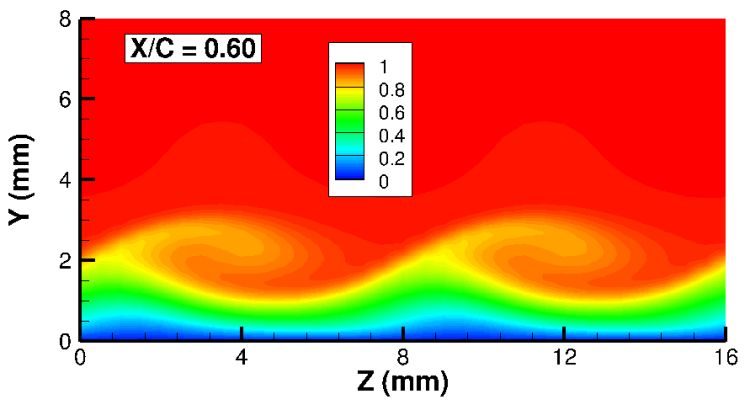

(c) $X / C=0.6$

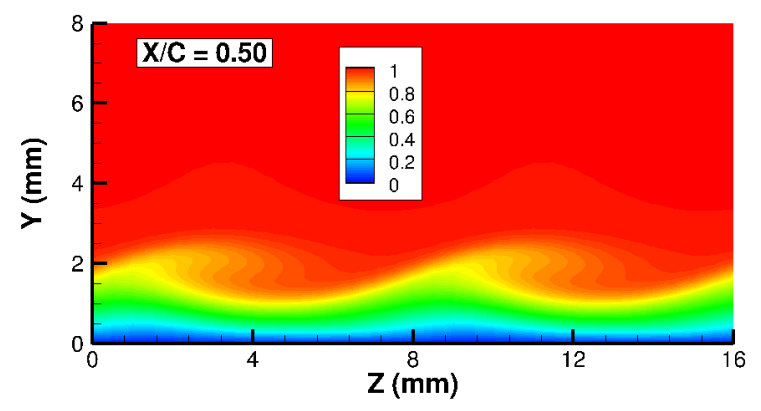

(b) $X / C=0.5$

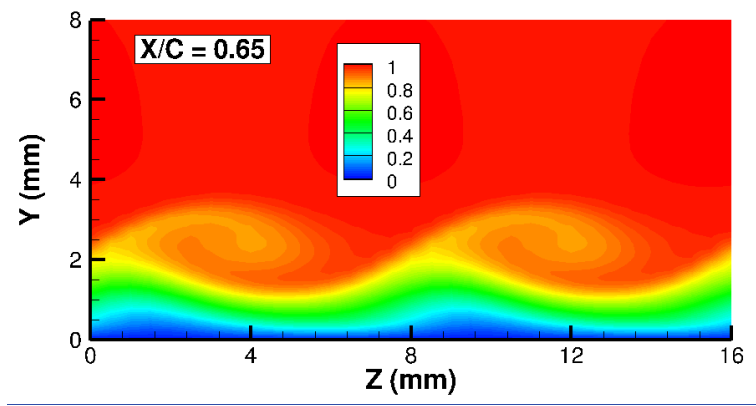

(d) $X / C=0.65$

Figure 2. Mean chordwise velocity contours for baseline flow with zero modulation (Case B).

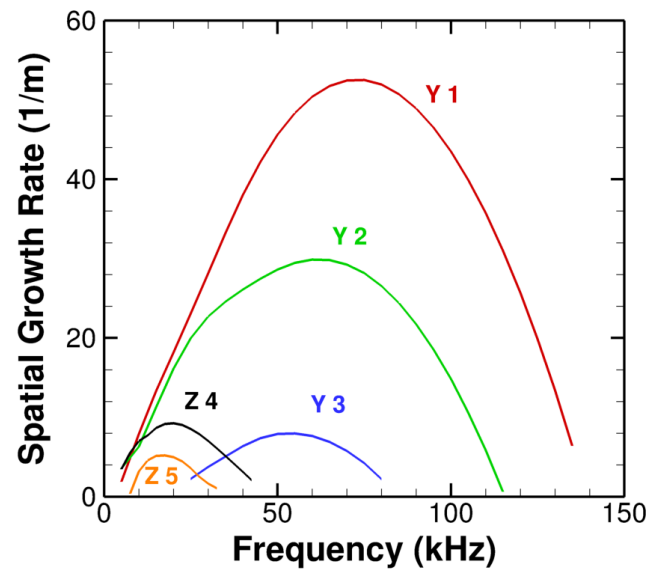

(a) Spatial growth rates.

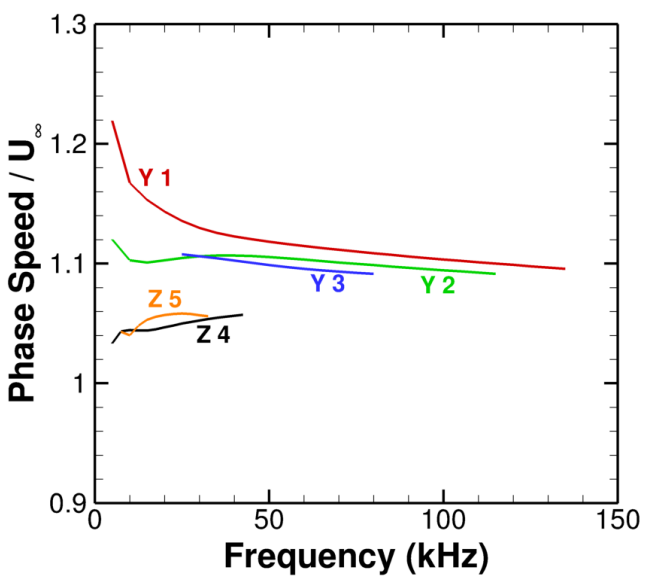

(b) Phase speeds.

Figure 3. Growth rates and phase speeds of the dominant families of secondary instability modes with fundamental spanwise wavelength at the location $X / C=0.6$ for case $B$. The curve legend indicates the dominant basic state shear ( $\mathrm{Y}$ or $\mathrm{Z}$ ) driving the growth of the secondary disturbance. 
The results of secondary instability analysis in Figs. 3(a) and (b) indicate the presence of at least five unstable modes at $X / C=0.60$. The spatial growth rates of these modes are plotted in Fig. 3(a), and Fig. 3(b) illustrates the corresponding phase speeds normalized by the freestream velocity. Three of the five modes correspond to the $\mathrm{Y}$ type of secondary disturbance, whose energy production mechanism is primarily related to the wall-normal shear associated with the basic state in Fig. 2. The remaining two modes are of the $\mathrm{Z}$ type, which derive a majority of their energy growth from the spanwise shear of the basic state. In some cases, secondary modes with comparable contributions to the overall enrgy production from both wall-normal and spanwise shear (i.e., YZ modes) have also been encountered $;^{18}$ however, none were found in this particular case. The fundamental mode of the $Y$ type has the highest growth rate in this baseline case. Figure 3(b) shows that the Y-mode secondary disturbances have higher phase velocities than the $\mathrm{Z}$ mode and the dominant $\mathrm{Y}$ mode (Mode $\mathrm{Y} 1$ ) also has the highest phase velocity among the secondary modes of interest. The phase velocities for most of the Y-mode disturbances are very close to each other and their frequency bandwidth tends to be significantly larger than that of the most unstable $\mathrm{Z}$ modes.

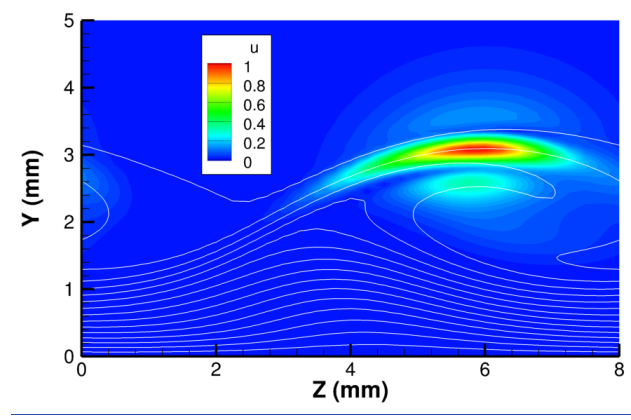

(a) Mode Y1.

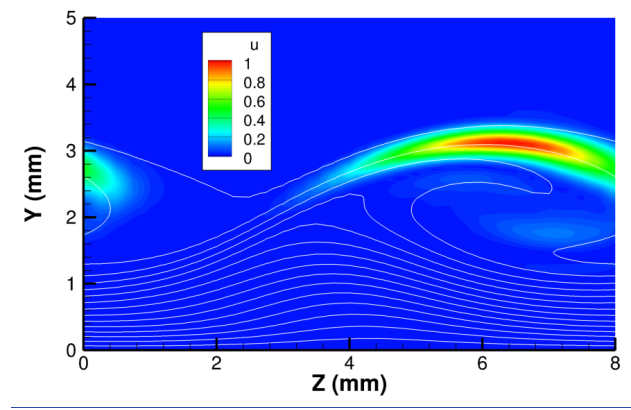

(c) Mode Y3.

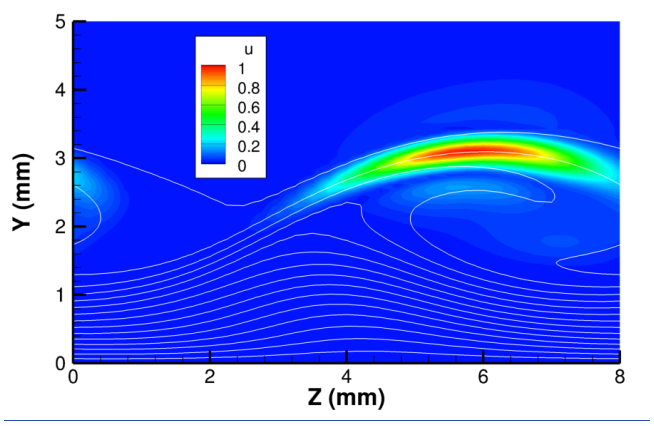

(b) Mode Y2.

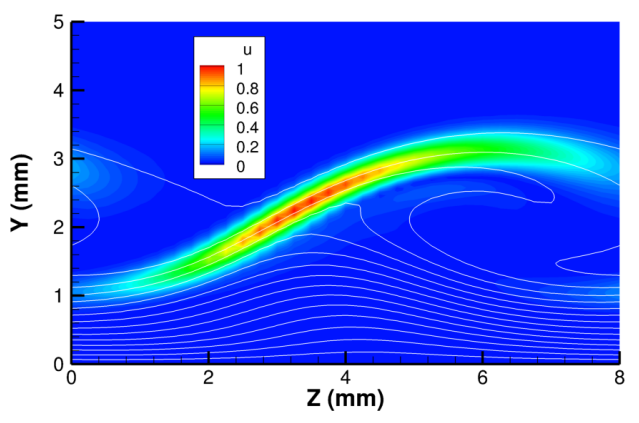

(d) Mode Z4.

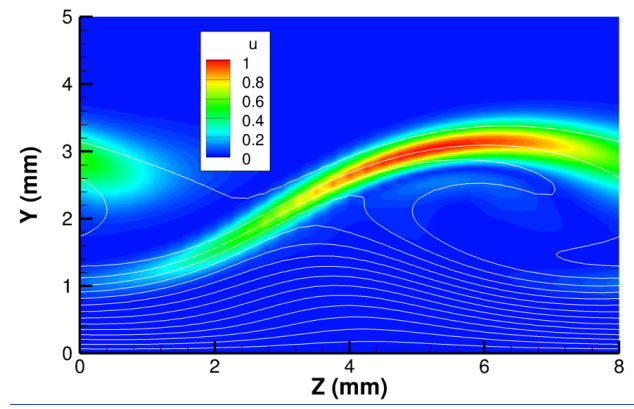

(e) Mode Z5.

Figure 4. Mode shapes in terms of the magnitude of chordwise velocity fluctuation for Y modes 1 through 3 (ranked within respective families in the order of decreasing peak growth rate in Fig. 3(a)) and the two Zmode secondary instabiliies at $X / C=0.60$. A single spanwise wavelength of the stationary crossflow vortex with $\lambda=8 \mathrm{~mm}$ is shown in the figure. 
Figures 4(a) and 4(b) reveal that both of the dominant Y modes (modes Y1 and Y2) are concentrated near the top of the vortex structure, but mode Y1 also has weaker secondary peaks above and below the primary peak. Mode Z4 represents the more unstable mode out of the two $\mathrm{Z}$ modes and is concentrated along the inclined portion of the shear layer that bounds the vortex structure (Fig. 4(d)). Somewhat surprisingly, the subdominant mode Z5 has peak fluctuations closer to the top of the vortex structure (Fig. 4(d)), yielding a mode shape that appears to resemble the mode shapes of both $\mathrm{Y}$ and $\mathrm{Z}$ modes.

To quantify the overall amplification potential of the above secondary modes, N-factor predictions for the dominant secondary disturbances in the baseline case are shown in Fig. 5. Only the Y1 and Y2 modes reach a peak $\mathrm{N}$ factor in excess of 6 , with mode $\mathrm{Y} 1$ reaching $\mathrm{N}=10$ near $X / C=0.60$. The highest $\mathrm{N}$ factor achieved by mode $\mathrm{Z} 4$ corresponds to $\mathrm{N} \approx 6$.

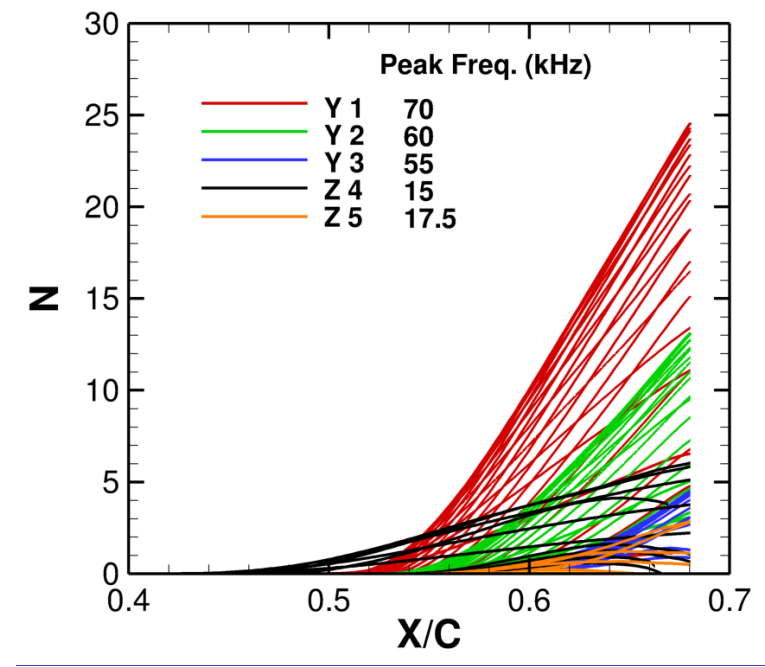

Figure 5. Chordwise evolution of linear $\mathrm{N}$-factors for Case B. Mode type and peak frequency in $\mathrm{kHz}$ is indicated in plot legend.

\section{Secondary Instability for Low Modulation Amplitude (case L)}

Figures $\underline{6}$ (a) through $\underline{6}$ (d) show the crossplane contours of normalized chordwise velocity at selected stations for Case $\mathrm{L}$. The nonlinear evolution of the stationary crossflow instability is visibly dominated by the $\lambda=8 \mathrm{~mm}$ mode, yet the low level of modulation leads to small yet noticeable differences between each adjacent pair of vortex structures corresponding to $\lambda=8 \mathrm{~mm}$. Out of these two vortex structures within each wavelength of the $\lambda=16 \mathrm{~mm}$ modulation, the left structure is somewhat stronger than the right one as seen from the stronger exchange of momentum within the crossplane (Fig. 2(d)). The difference between the strengths of the left and right vortex structures is presumably related to the nature of local interference between the $\lambda=8 \mathrm{~mm}$ and $\lambda=16 \mathrm{~mm}$ modes. Overall, the strongly overturned contours in Figs. $\underline{6}$ (c) and $\underline{6}$ (d) are similar to those for the baseline case, and hence, indicate a likely onset of comparable high-frequency secondary instability in Case L.

The secondary instability growth rates in Fig. 7 (a) and the associated mode shapes in Fig. 8 are consistent with the expectation that the more unstable secondary modes would be driven by the stronger of the two vortices within each modulating wavelength. Somewhat surprisingly, however, one also observes that all secondary modes are concentrated within only one or the other of the two vortices. Thus, if the modes confined to the left and right vortex structures were to be excited independently of each other (i.e., with zero correlation between them), then there would be little correlation between the fluctuations within the adjacent pair of $\lambda=8 \mathrm{~mm}$ vortices, despite the relatively weak modulation amplitude in this case.

The results of secondary instability analysis for the modulated stationary vortex pattern are shown in Figs. 7(a) and $\underline{7}(\mathrm{~b})$, and they indicate the presence of at least six unstable modes in Case $\mathrm{L}$. The spatial growth rates at $X / C=$ 0.6 are plotted in Fig. 7(a), and Fig. 7(b) illustrates the corresponding phase speeds. Four out of the six dominant modes correspond to the Y-type of secondary disturbance, whereas the remaining two are $\mathrm{Z}$ modes. Again, a fundamental wavelength mode of the Y-type has the highest growth rate in this case and the Y-mode secondary disturbances have higher phase velocities and larger frequency bandwidth than the $\mathrm{Z}_{-}$modes. 


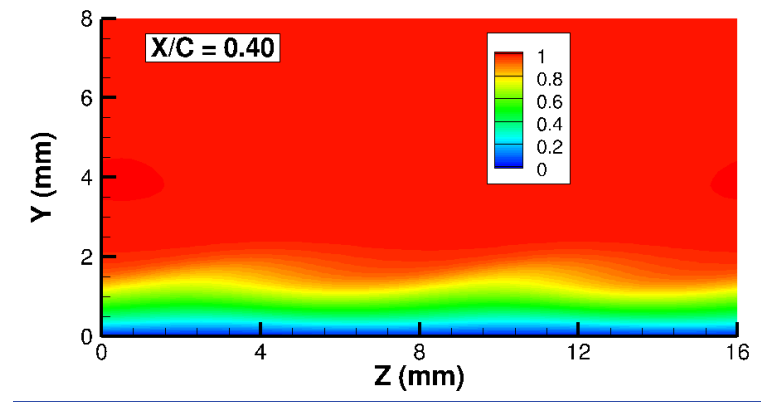

(b) $\underline{X / C}=\mathbf{0 . 3}$

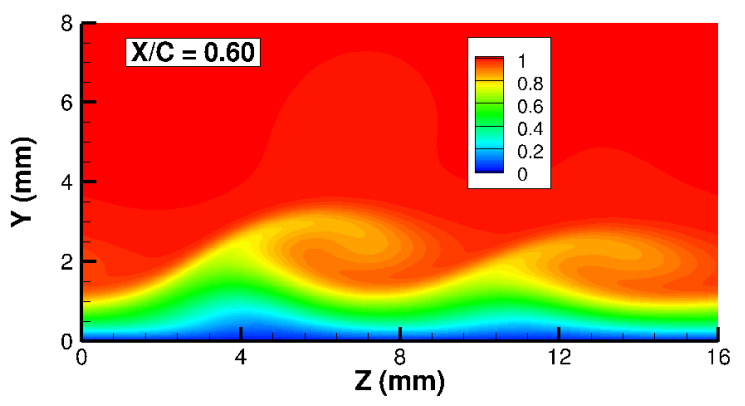

(c) $\underline{X / C}=0.5$

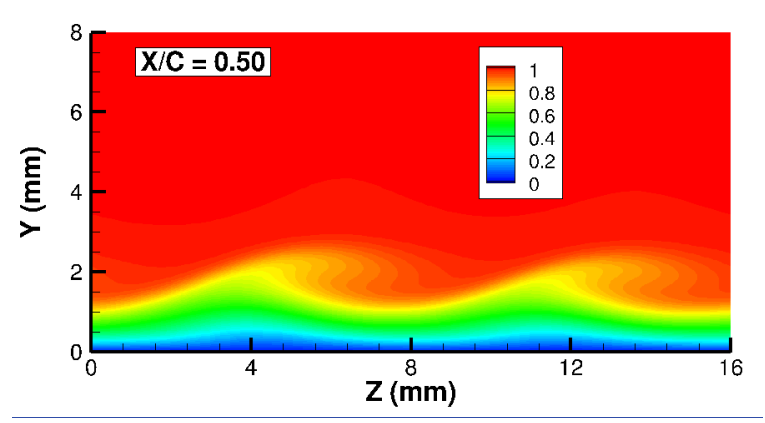

(b) $\underline{X / C}=0.4$

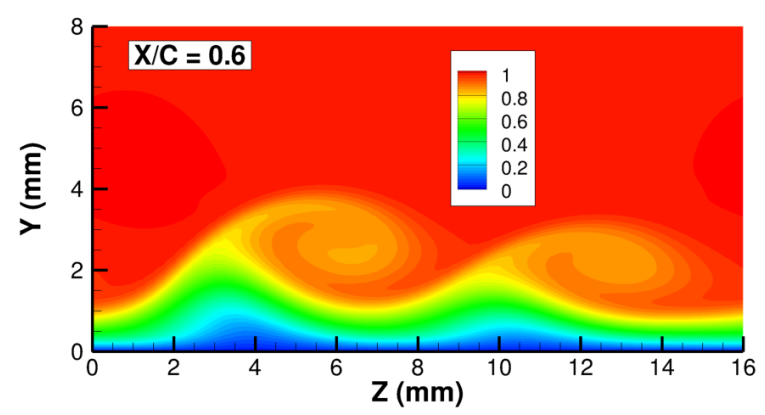

(d) $\underline{X / C}=\mathbf{0 . 6}$

Figure 6. Mean chordwise velocity contours for low modulation amplitude (Case L)

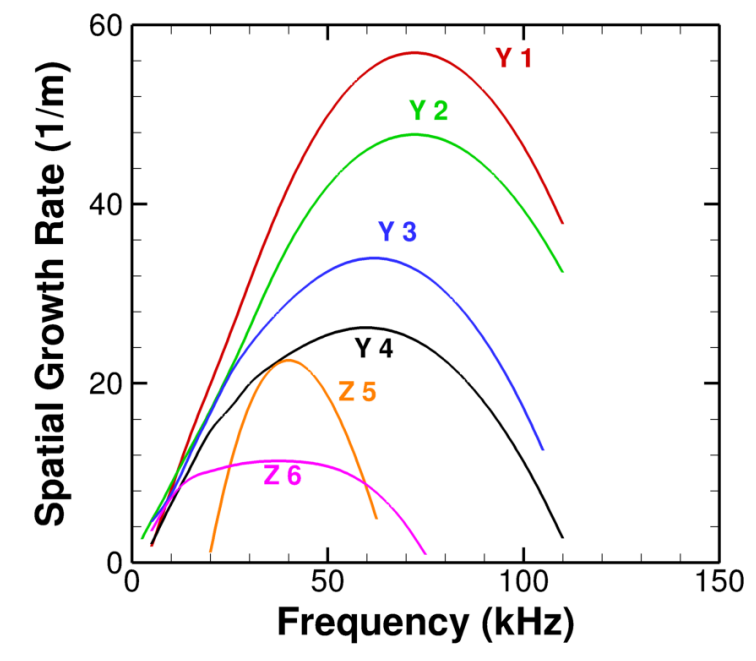

(a) Spatial growth rates.

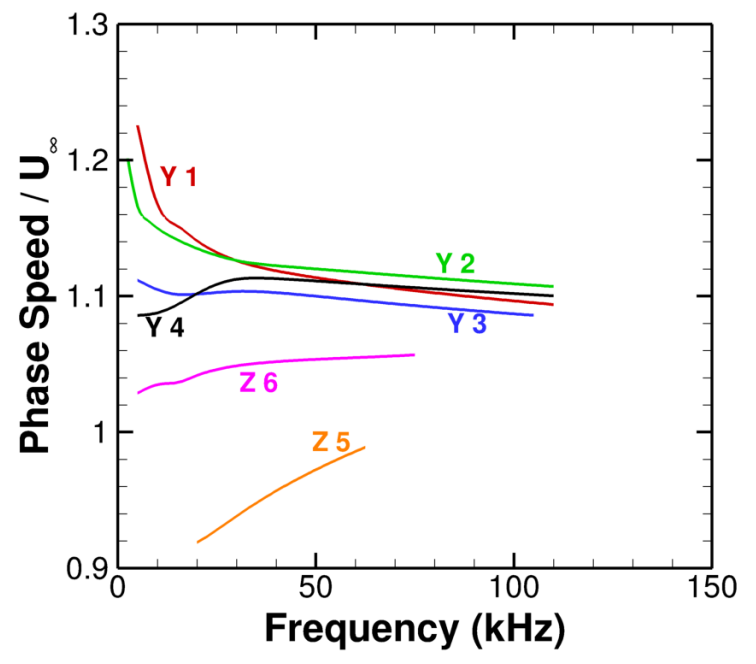

(b) Phase speeds.

Figure 7. Growth rates and phase speeds of the dominant families of secondary instability modes at $X / C=0.6$ for low modulation case $(\mathrm{L})$. The curve legend indicates the dominant basic state shear (Y or $\mathrm{Z}$ ) driving the growth of the secondary disturbance.

Illustrative mode shapes depicting the cross-sectional distribution of the chordwise velocity fluctuation associated with the different secondary mode disturbances are shown in Figs. $\underline{8}$ (a) through $\underline{8}(\underline{f})$. Inspection of these mode shapes indicates that the Y-modes of secondary instability supported by the modulated crossflow pattern are generally grouped in pairs, with each pair being linked to one of the dominant Y modes in the baseline case. The two modes within each pair have very similar mode shapes, but they reside on different vortex structures within the 
subharmonic wavelength. The Y-mode disturbance that is concentrated in the stronger, leftward structure corresponds to the higher growth rate within each pair of modes. Indeed, the peak growth rates of both modes within a pair bracket the peak growth rate of the corresponding Y mode in the baseline case, presumably because the strength of the Y-mode secondary instability is related to the basic state shear within the vortex structure and the influence of modulation on this shear. Thus, effectively, the modulation causes each mode in the baseline case to split into a pair of modes. In the absence of modulation, any pair of adjacent crossflow vortices involves identical vortex structures, and hence, the two secondary modes reduce to the single mode seen in Case B. The most interesting part of the mode shapes in Fig. 4 is that each mode shape is primarily concentrated in only one of the two vortex structures across the $16 \mathrm{~mm}$ wavelength of the modulating disturbance. In other words, regardless of the mode type, neither of the secondary modes has a signature that extends over the entire wavelength of the subharmonic stationary mode. In contrast to the Y-mode pairs, however, the two Z modes (Figs. $\underline{8}(\mathrm{e})$ and $\underline{8}(\mathrm{f})$ ) are concentrated solely within the stronger, leftward vortex structure. They do not appear to have significantly unstable counterparts that reside on the weaker vortex structure on the right hand side of these figures. Thus, the modest, yet finite amplitude modulation in Case L already has a stronger and more complex influence on the Z-mode instability.

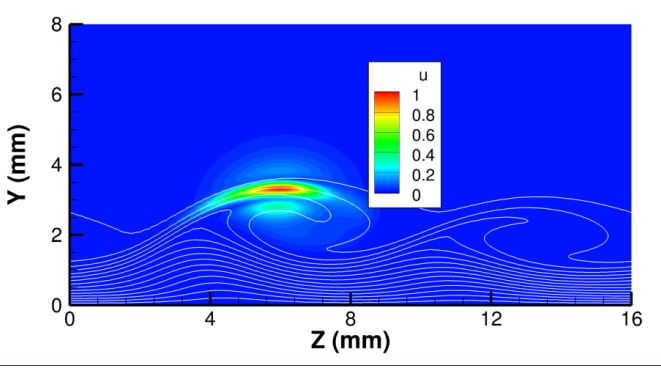

(a) Mode Y1.

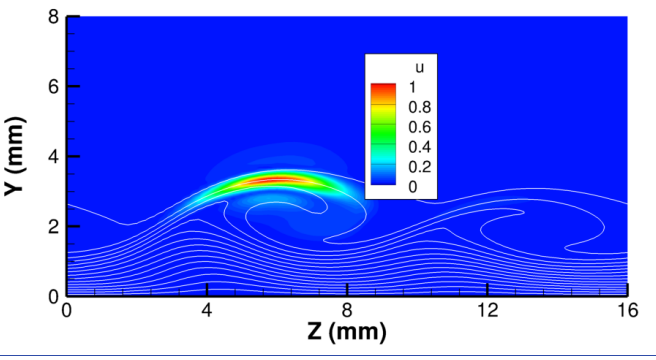

(c) Mode Y3.

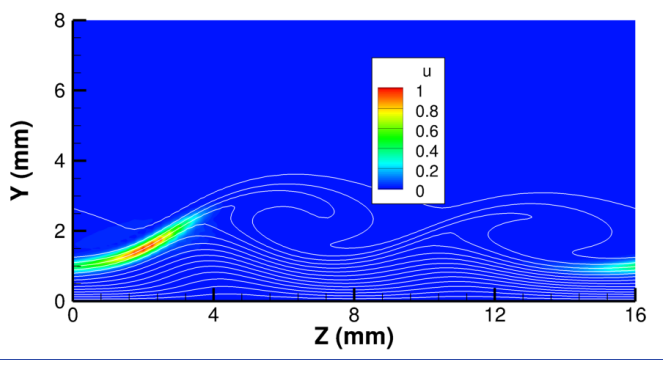

(e) Mode Zz5.

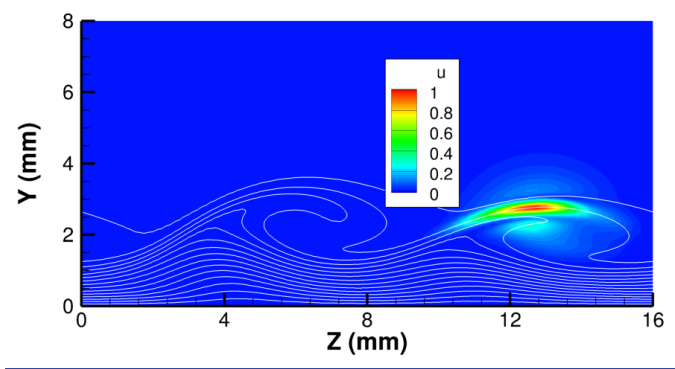

(b) Mode Y2.

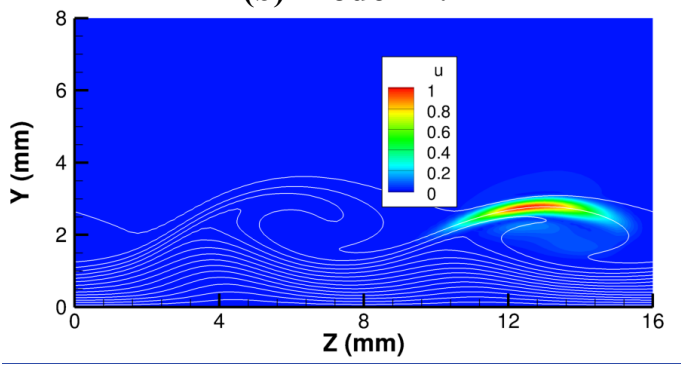

(d) Mode Y4.

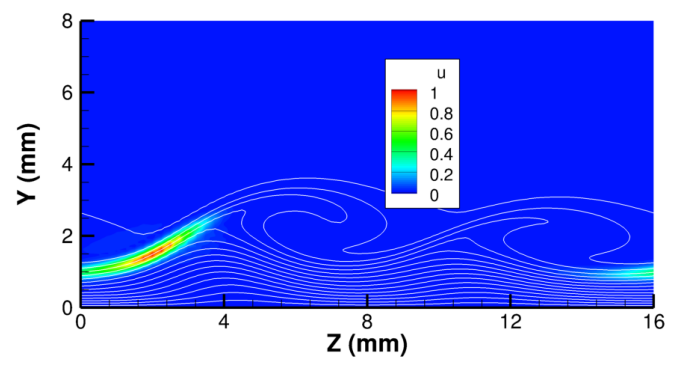

(f) Mode Z6.

Figure 8. Mode shapes in terms of the magnitude of chordwise velocity fluctuation for Y-modes 1 through 4 (ranked by decreasing order of peak growth rate in Fig. 3(a)) and the two Z-modes of secondary instability at $X / C=0.60$. Two spanwise wavelengths of the stationary crossflow vortex with $\lambda=8 \mathrm{~mm}$ are shown in each figure.

To quantify the overall amplification potential of the secondary disturbances, N-factor predictions for the dominant secondary disturbances of various selected frequencies are shown in Fig. 9 . Results from Fig. 9 show that all four Y modes reach $\mathrm{N}$ factors well in excess of 10, and hence, have the potential to produce transition on their own. Furthermore, the presence of modulation has strengthened the $\mathrm{Z}$ mode of instability so that mode $\mathrm{Z} 5$ reaches 
$\mathrm{N} \approx 8$ and may be able to cause transition by itself as well. Consistent with the peak growth rates at $X / C=0.60$ in Fig. 7(a), the peak $\mathrm{N}$ factors of modes $\mathrm{Y} 1$ and $\mathrm{Y} 2$ in Case L bracket the peak $\mathrm{N}$ factor of the Y1 mode in the baseline case, and the $\mathrm{N}$ factors of modes $\mathrm{Y} 3$ and $\mathrm{Y} 4$ in Case L bracket the Y2 mode $\mathrm{N}$ factors in the baseline case.

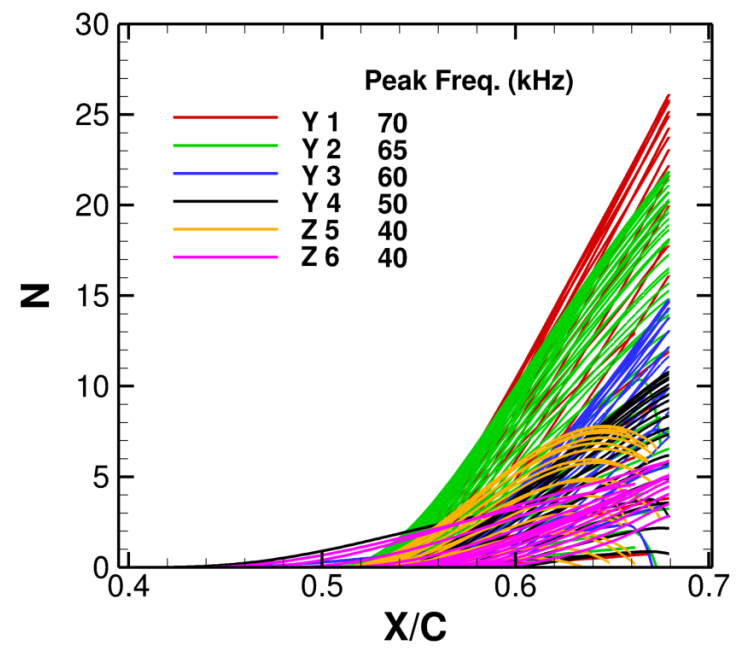

Figure 9. Chordwise evolution of linear N-factors for Case L. Mode type and peak frequency in $\mathrm{kHz}$ is indicated in plot legend.

\section{Secondary Instability for Case H}

The case of highest modulation amplitude is considered next. The increased amplitude of the $\lambda=16 \mathrm{~mm}$ mode leads to a greater disparity between the strengths of the two vortex structures within each modulating wavelength at $X / C=0.5$ (Fig. 10(a)). In fact, the $\lambda=16 \mathrm{~mm}$ mode becomes dominant over a significant region of the computation. Thus, at $X / C=0.6$ (Fig. 10(b)), the stronger vortex on the left appears to have nearly engulfed the weaker vortex, and at $X / C=0.6$ (Fig. 10(c)), the vortex pattern displays a single dominant vortex across the $16 \mathrm{~mm}$ wavelength.

The secondary instability characteristics at $X / C=0.60$ are summarized in Figs. 11(a)-(b) and 12(a)-(e). The number of significant unstable modes is now reduced by one in comparison with the case of weaker modulation in Section III-B; and more importantly, no distinct pairing of the mode shapes is observed in this case. The absence of mode splitting is attributed to the dominance of the subarhmonic mode that leads to a single prominent vortex structure across the subharmonic wavelength of $\lambda=16 \mathrm{~mm}$. Furthermore, the Z-mode instability has become significantly stronger with the increased amplitude of modulation so that the maximum growth rate of mode Z2 is now larger than that of mode Y3 and approaches the peak growth rate of the most unstable mode Y1 at this location. 


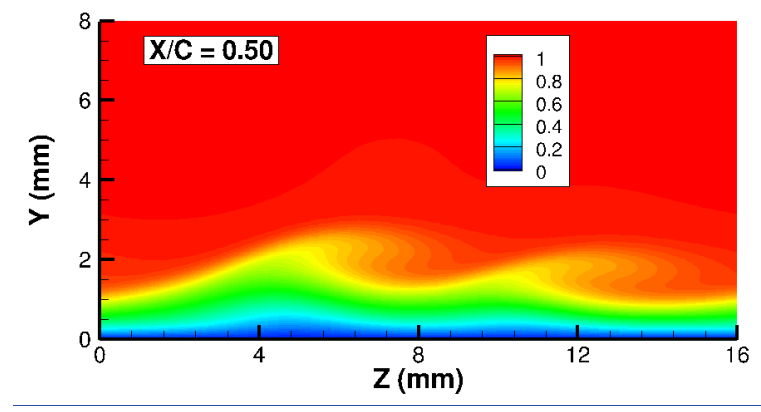

(a) $\underline{X / C}=\mathbf{0 . 5}$

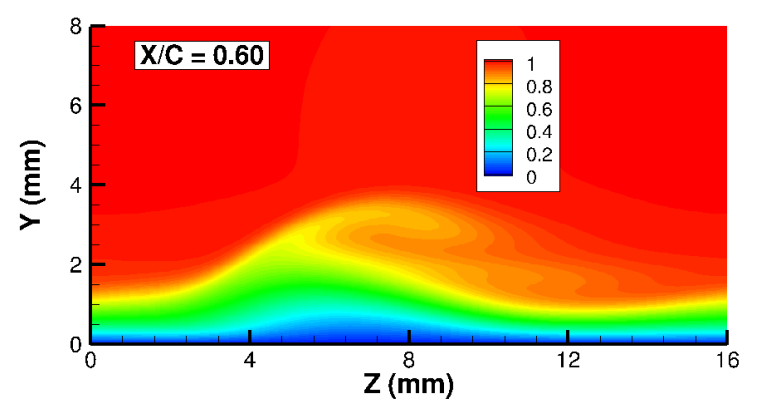

(b) $\underline{X / C}=\mathbf{0 . 6}$

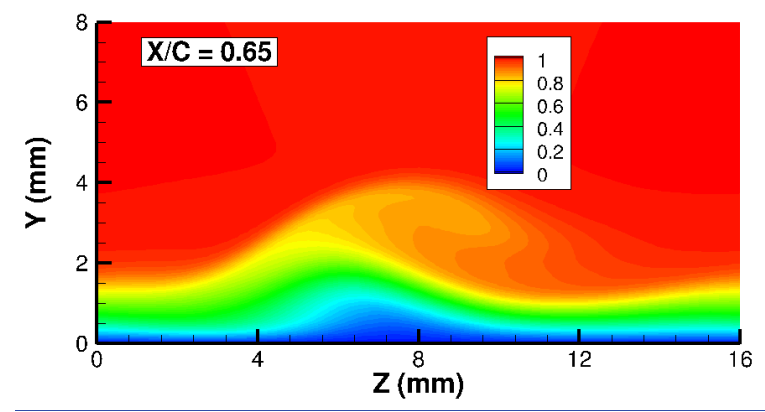

(c) $X / C=0.65$

Figure 10. Mean chordwise velocity contours for high modulation amplitude (Case $\mathrm{H})$.

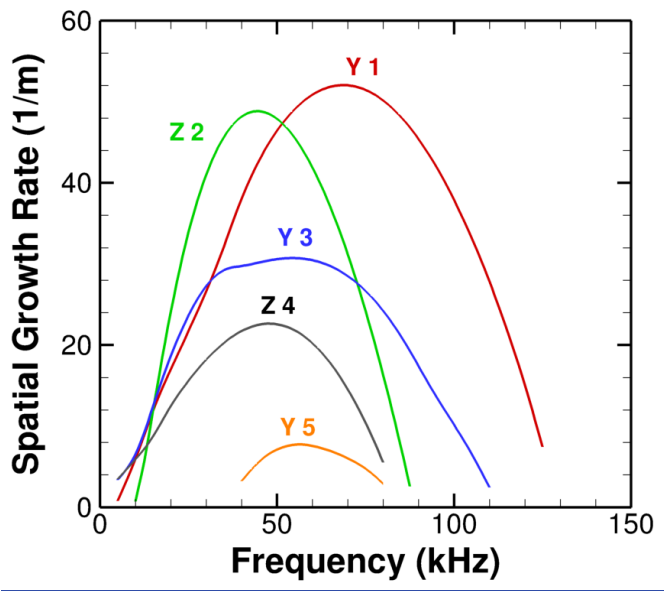

(a) Spatial growth rates.

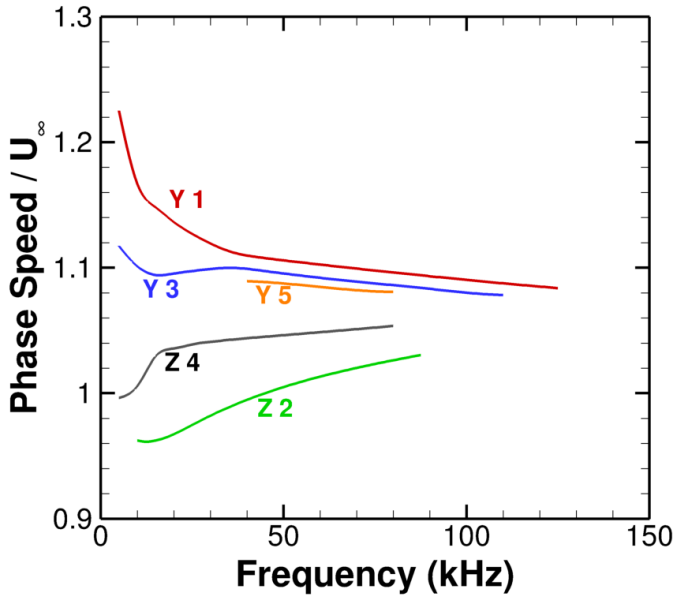

(b) Phase speeds.

Figure 11. Growth rates and phase speeds of the dominant families of secondary instability modes at $X / C=0.60$ for high modulation amplitude (Case $\mathrm{H})$. The curve legend indicates the dominant basic state shear ( $\mathrm{Y}$ or $\mathrm{Z}$ ) driving the growth of the secondary disturbance. 


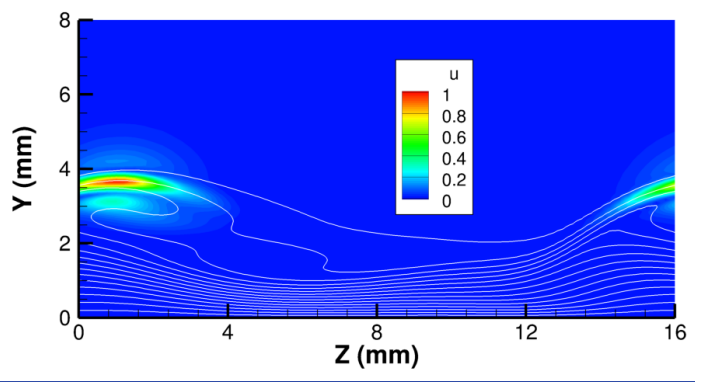

(a) Mode Y1.

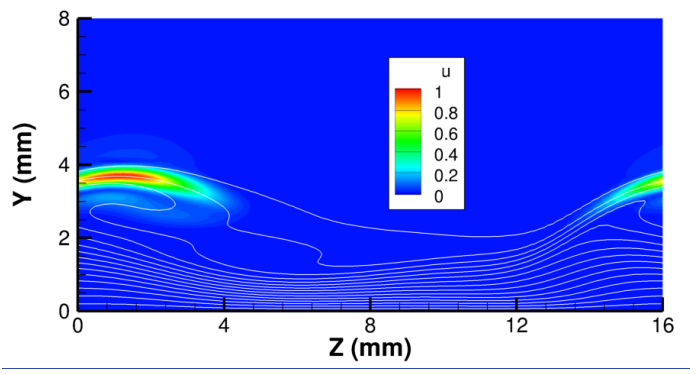

(c) Mode Y3.

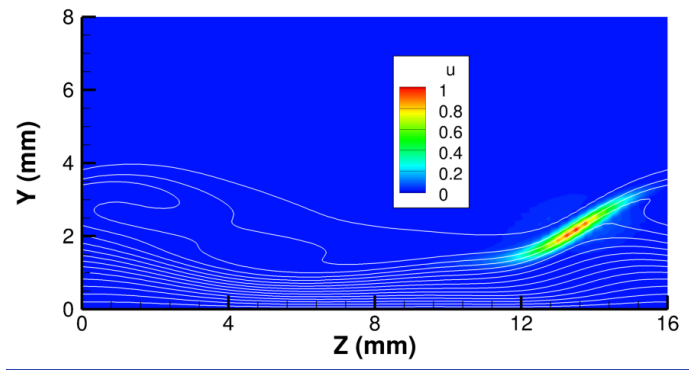

(b) Mode Z2.

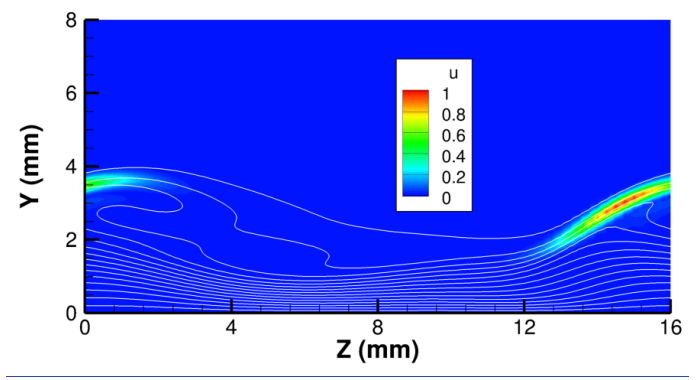

(d) Mode Z4.

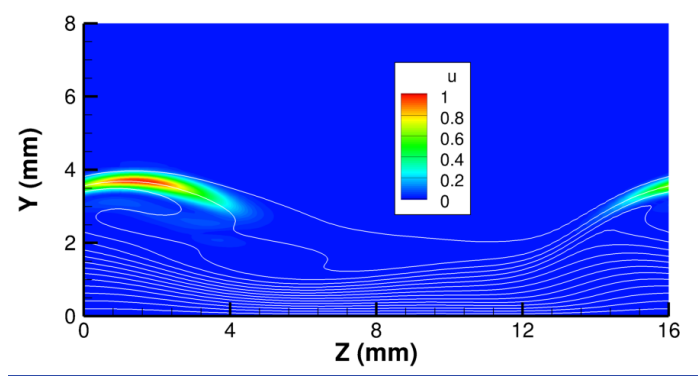

(e) Mode Y5.

Figure 12. $|\mathbf{u}|$ mode shapes for dominant modes of secondary instability at $X / C=0.60$ for highest amplitude of modulation (Case H). The horizontal extent of each figure corresponds to the wavelength of the modulating mode (i.e., $16 \mathrm{~mm}$ ).

The $\mathrm{N}$-factor evolution in Fig. 13 is consistent with the previously noted trends in local amplification rates at $X / C$ $=0.60$ (Fig. 11(a)). Thus, the highest $\mathrm{N}$ factor reached by mode $\mathrm{Z} 2$ has now increased to 25 , which is in fact larger than the peak Y-mode $\mathrm{N}$ factor of 22 because of the earlier onset of $\mathrm{Z} 2$ mode amplification. 


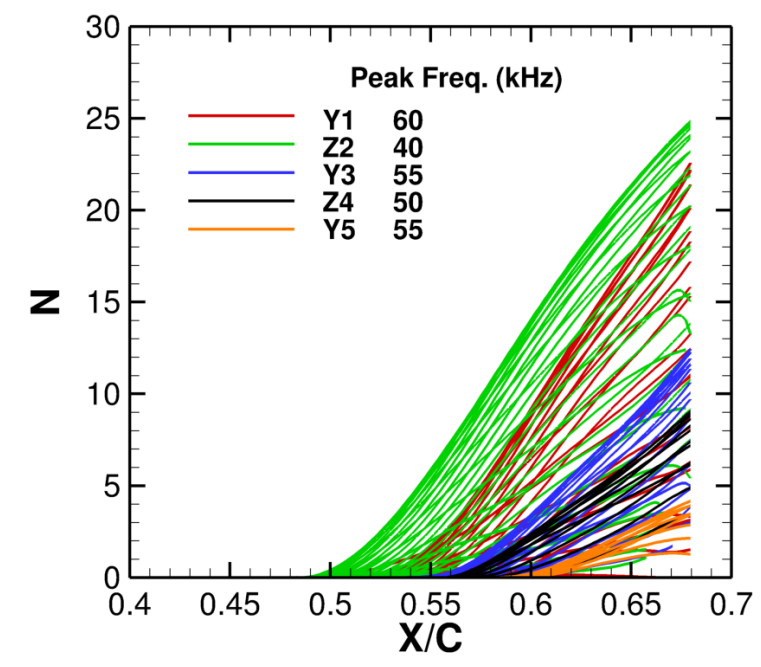

Figure 13. Chordwise evolution of linear $\mathbf{N}$-factors for high modulation amplitude (Case $\mathbf{H}$ ). Mode type and peak frequency in $\mathrm{kHz}$ is indicated in plot legend.

\section{E. Secondary Instability for Case M}

Case $\mathrm{M}$ corresponds to an intermediate value of the modulation amplitude in relation to the two cases previously considered in subsections III-C and III-D above. As expected, the modal characteristics in Figs. 15(a)-(b) appear to fall in between those of Case L than Case H. However, because of the presence of two distinct vortex structures across the subharmonic wavelength (Figs. 14(a)-(b)), the mode shapes in Figs. 16(a)-(h) are slightly more similar to those in Case L than in Case H. Inspection of the mode shapes provides partial evidence of mode splitting. Thus, the two dominant Y modes (modes Y1 and Y3) and both Z modes (modes Z2 and Z4) are concentrated along the stronger vortex on the left and have relatively weak fluctuations along the vortex on the right, whereas the subdominant mode Y5 has peak fluctuations along the weaker vortex on the right. On the other hand, the greater disparity between the left and right vortex structures than that in Case L leads to larger differences among the overall growth rates of the $\mathrm{Y}$-modes. Consequently, no pairwise grouping is obvious among the $\mathrm{Y}$ modes in Case M. Figure 17 confirms the stronger effect of the stationary modulation on the amplification of the Z-mode, which now reaches a peak $\mathrm{N}$ factor of nearly 18 . Even though the peak $\mathrm{N}$ factor of the $\mathrm{Z} 2$ mode is lower than that of the Y1 mode, the $\mathrm{Z}$ mode reaches the intermediate $\mathrm{N}$-factor value of 10 before the $\mathrm{Y} 1$ mode does, and hence, mode $\mathrm{Z} 2$ could well be the dominant catalyst for transition even in Case M.

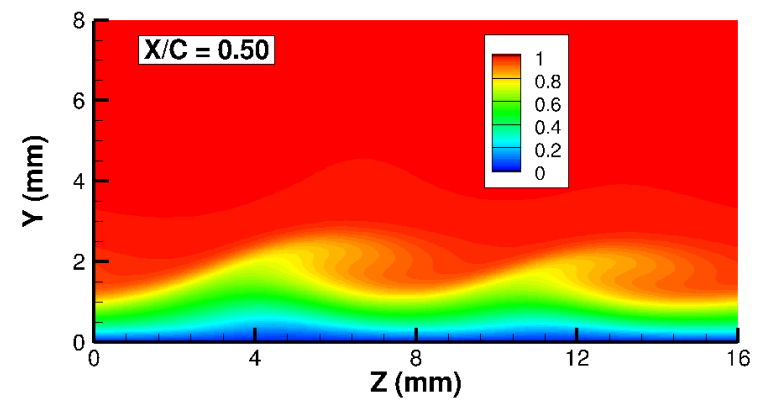

(a) $\underline{X / C}=\mathbf{0 . 5}$

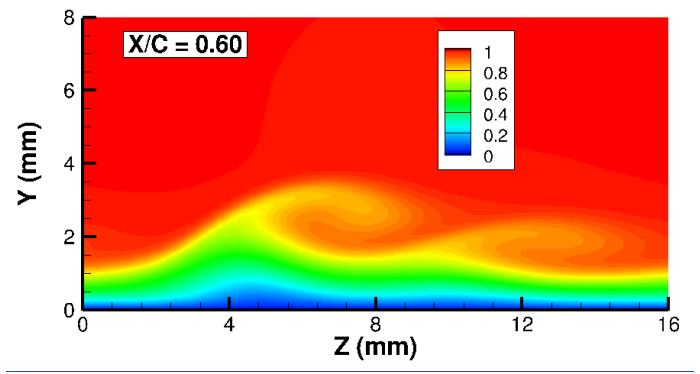

(b) $\underline{X / C}=\mathbf{0 . 6} \underline{0}$

Fig. 14. Mean chordwise mean velocity contours for mid level modulation (Case M). 


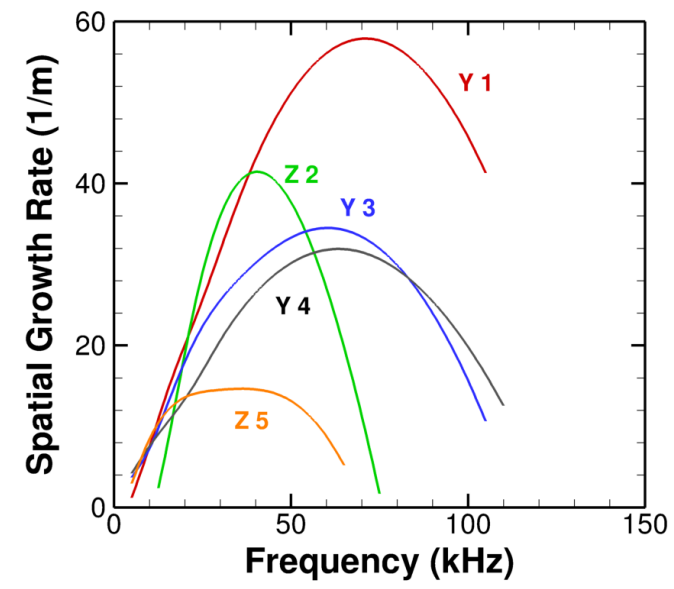

(a) Spatial growth rates.

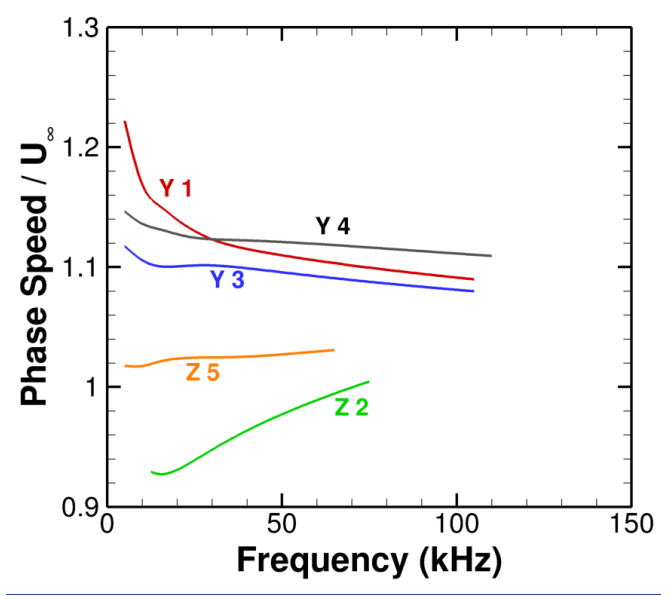

(b) Phase speeds.

Figure 15. Spatial growth rates and phase speeds of the dominant families of secondary instability modes at $X / C=0.6$ for medium modulation amplitude (Case $M$ ). The curve legend indicates the dominant basic state shear (Y or $\mathrm{Z})$ driving the growth of the secondary disturbance.

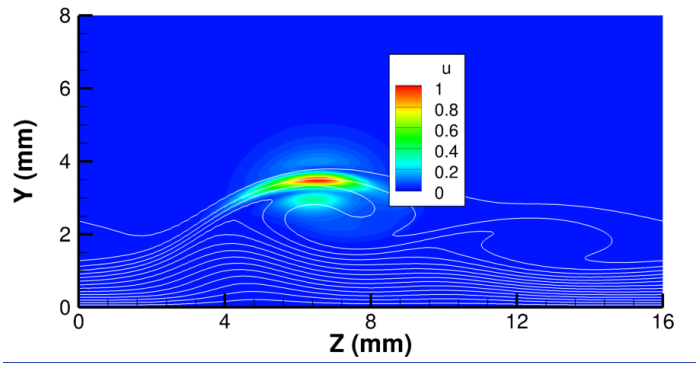

(a) Mode Y1.

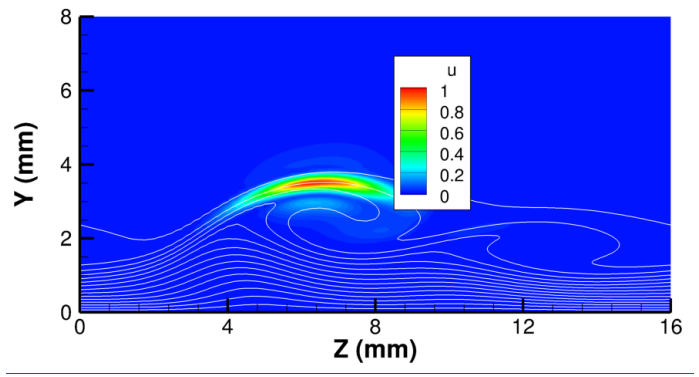

(c) Mode Y3.

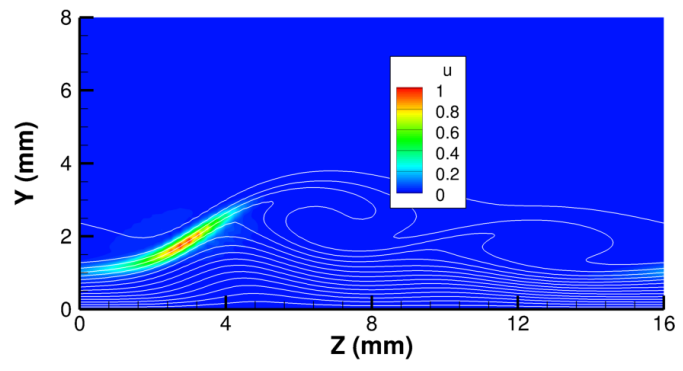

(b) Mode Z2.

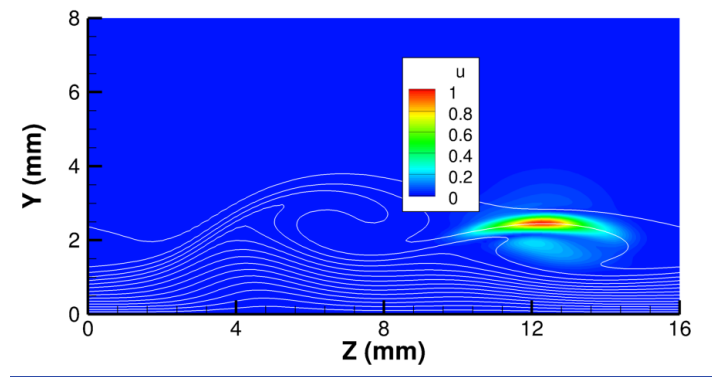

(d) Mode Y4.

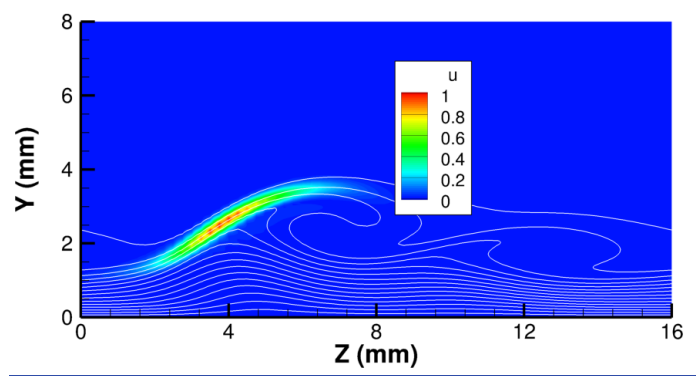

(g) Mode Z5.

Figure 16. $|\mathbf{u}|$ mode shapes at $X / C=0.6$ for medium modulation amplitude (Case M). Two spanwise wavelengths of the stationary crossflow vortex with $\lambda=8 \mathrm{~mm}$ are shown in the figure. 


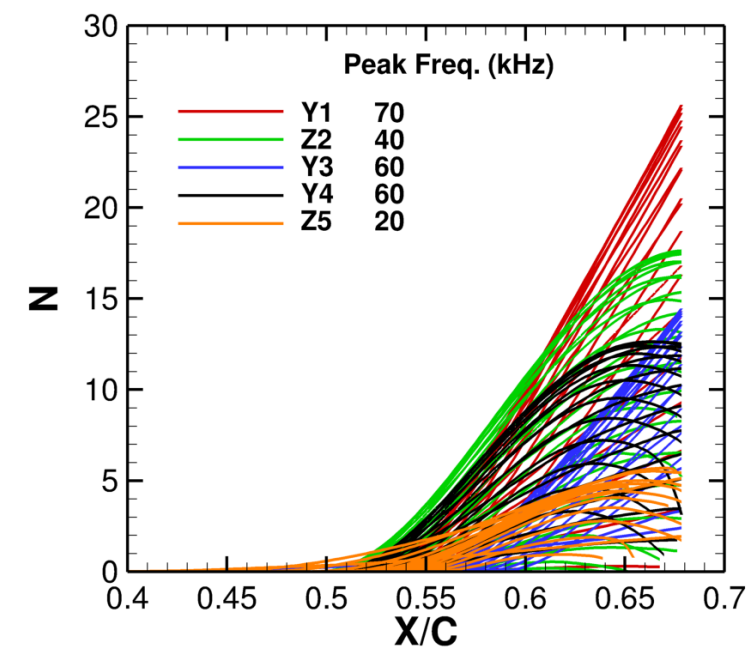

Figure 17. Chordwise evolution of linear N-factors for mid level modulation (Case M). Mode type and peak frequency in $\mathrm{kHz}$ is indicated in plot legend.

\section{Nonlinear Evolution of Secondary Instability in the Presence of Modulation}

Next, we explore the question of whether and how the presence of the modulating component influences transition onset due to nonlinear breakdown of the secondary instability modes described in the previous section. To that end, NPSE computations were performed for two selected cases from Section III, involving a Y-mode disturbance and a Z-mode disturbance, respectively. These results extend the previous findings ${ }^{9-11}$ for $\mathrm{Y}-$ and $\mathrm{Z}-$ mode breakdowns in flow configurations without any modulation. The amplitude evolution during the Z-mode breakdown is shown in Fig. 18, where we have plotted the RMS amplitude of the fundamental frequency associated with the secondary instability (denoted as $\mathrm{m}=1$ ) and its various harmonics $(\mathrm{m}=2-5)$ as functions of the chordwise coordinate. At each chordwise location, the maximum of the RMS fluctuation over the cross-section of the vortex pattern is chosen as a measure of the local amplitude.

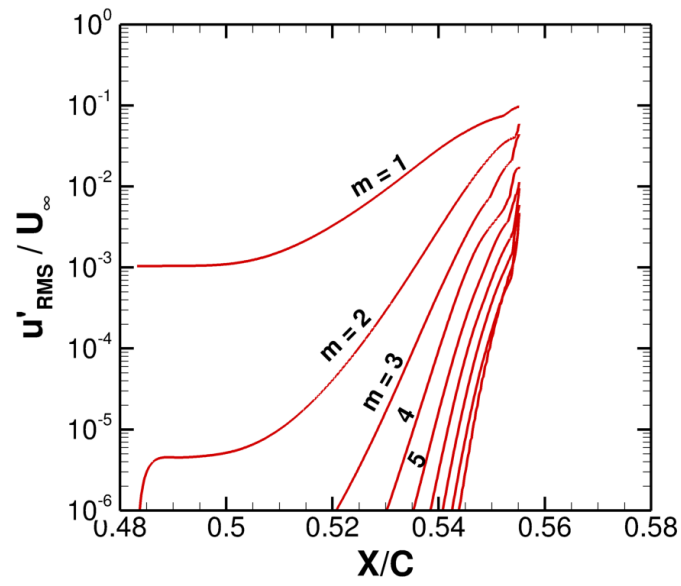

Figure 18. Nonlinear evolution of amplitudes during the nonlinear breakdown of Z2-mode disturbance with frequency of $30 \mathrm{kHz}($ Case $\mathrm{H})$. Normalized initial amplitude of the $\mathrm{Z2}$ mode at $X / C=0.485$ is $A_{\text {init }}=$ $\underline{10^{-3}}$. 
In the entire region of this computation, which extends somewhat past the onset of rise in skin friction near $X / C=$ 0.552 , the fundamental harmonic $(\mathrm{m}=1)$ remains the most energetic among all modes. Similar to the $\mathrm{Z}$-mode breakdown case in Refs. [9] and [11], the fundamental harmonic grows linearly until the stabilizing influence of nonlinearity causes the growth rate to decrease relative to its initial, weakly nonlinear evolution. Following the region of reduced growth rate, the fundamental amplitude begins to increase more rapidly in the vicinity of the transition onset location. The amplitude of the $\mathrm{m}=3$ harmonic displays a similar pattern of decreased and increased growth rate as the fundamental $(\mathrm{m}=1)$, but the changes in its evolution appear to lead those in the fundamental. The evolution of the $m=4$ and $m=5$ harmonics displays two different episodes of this stair-stepping structure. The rapid increase in modal amplitude during the second episode corresponds to a significantly higher growth rate than that during the initial, weakly nonlinear evolution, and hence, is suggestive of a strong tertiary instability that amplifies over a shorter spatial scale. While the rapid growth slows down after the amplitudes of the $m=4$ and $m=5$ harmonics have increased sufficiently, the increased amplitudes of those modes appear to induce a reverse energy cascade involving a rapid rise in the amplitudes of successively lower harmonics. An analogous cascade was also seen during the Z-mode breakdown in the baseline case. ${ }^{11}$

Figure 19 shows the cross-plane distributions of RMS perturbation associated with the high frequency instability at selected chordwise locations. At each station, the amplitude distribution is normalized to have a maximum value of unity. At $X / C=0.501$, the amplitude distribution mimics the linear eigenfunction of the $\mathrm{Z} 2$ mode from Fig. 12(b); and it retains that similarity until very close to the transition onset location. Just before breakdown, the fluctuations concentrated along the inclined shear layer develop a localized distortion near the location of peak RMS amplitude, which leads to the development of unsteady structures with smaller spanwise scales. Farther downstream, the amplitude distributions begin to spread out, thus affecting a larger cross-sectional area of the merged vortex structure. The overall development of the RMS velocity fluctuations is quite similar to that in the zero modulation case. ${ }^{11}$

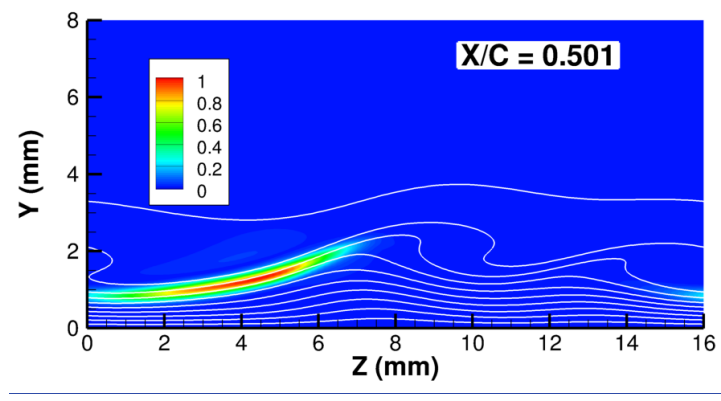

(a) $X / C=0.501$

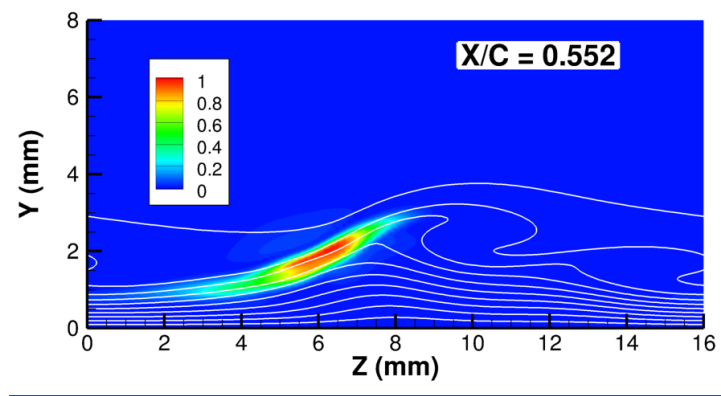

(c) $X / C=0.552$

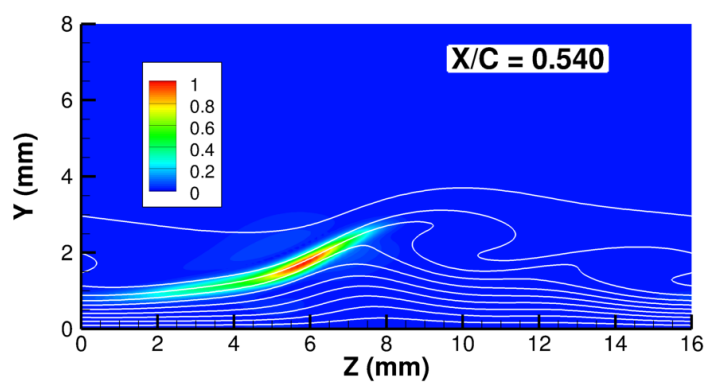

(b) $X / C=0.540$

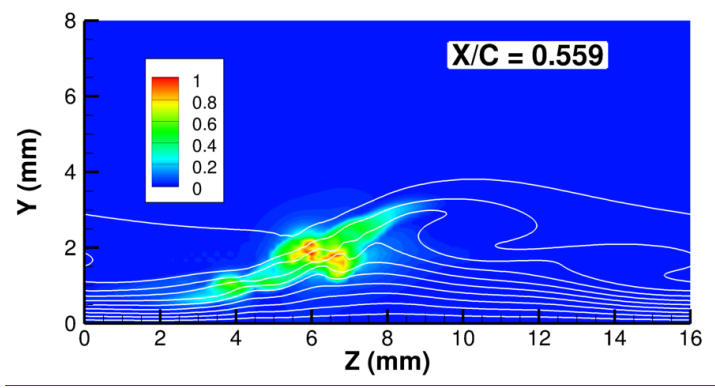

(d) $X / C=0.559$

Figure 19. Time-RMS distributions of chordwise velocity at selected chordwise stations during the nonlinear breakdown of Z2-mode disturbance in Case H (flow parameters are same as Fig. 18). The white lines in the background denote the chordwise velocity contours of unperturbed, stationary crossflow vortex.

During Z-mode breakdown in the absence of any modulation, ${ }^{11}$ the onset of transition was followed by a spanwise spreading of turbulence within a wedgelike region that is analogous to the problem of turbulent contamination. In the present case with a subharmonic modulation, the turbulence needs to spread across the 
modulation wavelength of $16 \mathrm{~mm}$ rather than across the $8 \mathrm{~mm}$ width of the dominant vortex structures in the zero baseline case. Assuming that the wedge angle corresponding to spanwise contamination remains approximately same (since it is likely to be determined by the local Reynolds number and Mach number), the transition zone in the presence of modulation is expected to be twice as long as that in the baseline case. However, numerical simulations are required to confirm this conjecture. Although results for the Y-mode breakdown case are not shown in this paper, the above remarks concerning the spanwise spreading of turbulence also apply to that transition scenario (see, also, Ref. 10 for DNS results pertaining to Y-mode breakdown in a zero modulation case).

\section{Summary}

In this paper, previously reported computations of secondary instability of stationary crossflow modes were extended from crossflow modes of a single spanwise wavelength to vortex patterns that are modulated in the spanwise direction by subharmonic stationary modes. Secondary instability of the modulated crossflow pattern is studied using planar, partial-differential-equation based eigenvalue analysis. Results show that modulation by the first subharmonic of the input stationary mode results in mode splitting that leads to a pair of secondary modes with somewhat different amplification rates and that are concentrated within different individual vortex structures across the subharmonic wavelength. The mode splitting phenomenon suggests that a more complex stationary modulation, such as that induced by natural surface roughness, would yield a considerably richer spectrum of secondary instability modes. Because of the spatial decoupling between the modes, the spanwise modulation should also enhance the decorrelation between secondary mode fluctuations across the spanwise pattern of stationary crossflow vortices.

Computations of the nonlinear development of selected secondary instability modes have shown that the essential features of nonlinear evolution in the modulated flow configurations are rather similar to those documented previously in the zero modulation case. The similarity in transition onset mechanisms may be attributed to the similarity in the mode shapes of the dominant secondary instabilities, which in both cases, are concentrated along specific subregions of the shear layer that bounds the stationary vortex structure. The main anticipated effect of modulation corresponds to an increased length of the transition zone, but numerical simulations are desirable to confirm that effect.

\section{Acknowledgments}

This work was performed as part of the Revolutionary Computational Aerosciences discipline under the Transformational Tools and Technologies project of NASA's Transformative Aeronautics Concepts Program. Resources supporting this work were provided by the NASA High-End Computing (HEC) Program through the NASA Advanced Supercomputing (NAS) Division at Ames Research Center.

\section{References}

${ }^{1}$ Kohama, Y., Saric, W. S., and Hoos, J. A., “A High-Frequency Secondary Instability of Crossflow Vortices that Leads to Transition," in Boundary Layer Transition and Control, Proc. Royal Aeronautical Society, Cambridge, 8-12 April, 1991, London, UK.

${ }^{2}$ Balachandar, S., Streett, C. L., and Malik, M. R., "Secondary Instability in a Rotating Disk Flow,” J. Fluid Mech., Vol. 242, 1992, pp. 323-347.

${ }^{3}$ Malik, M. R., Li, F., and Chang, C.-L., "Crossflow Disturbances in Three-Dimensional Boundary Layers: Nonlinear Development, Wave Interaction and Secondary Instability,” J. Fluid Mech., Vol. 268, 1994, pp. 1-36.

${ }^{4}$ Malik, M. R., Li, F., Choudhari, M., and Chang, C.-L., "Secondary Instability of Crossflow Vortices and Swept-Wing Boundary-Layer Transition,” J. Fluid Mech., Vol. 399, 1999, pp. 85-115.

${ }^{5}$ White E. B. and Saric W. S., "Secondary Instability of Crossflow Vortices,” J. Fluid Mech., Vol. 525, pp. 275-308, 2005.

${ }^{6} \mathrm{Li}$, F. and Choudhari, M., "Spatially Developing Secondary Instabilities and Attachment Line Instability in Supersonic Boundary Layers," AIAA Paper 2008-590, 2008.

${ }^{7}$ Carpenter, A. L., Saric, W. S., and Reed, H. L., "Laminar Flow Control on a Swept Wing with Distributed Roughness," AIAA Paper 2008-7335, 2008.

${ }^{8}$ Li, F., Choudhari, M. M., Chang, C.-L. Streett, C. L., and Carpenter, M. H., "Computational Modeling of Roughness-Based Laminar Flow Control on a Subsonic Swept Wing," AIAA J., Vol. 49, No. 3, March 2011, pp. 520-529.

${ }^{9}$ Li, F., Choudhari, M. M., Carpenter, M. H., Malik, M. R., Chang, C.-L. and Streett, C. L., "Roughness Based Crossflow Transition Control for a Swept Airfoil Design Relevant to Subsonic Transports," AIAA Paper 2010-4380, 2010.

${ }^{10}$ Duan, L., Choudhari, M., and Li, F., "Direct Numerical Simulation of Crossflow-Induced Transition in a Swept Wing Boundary Layer,” AIAA Paper 2013-2617, 2013. 
${ }^{11}$ Choudhari, M., Li, F., Duan, L., Carpenter, M. H., Streett, C. L. and Malik, M. R., "Towards Bridging the Gap in Holistic Transtion Prediction via Numerical Simulations," AIAA Paper 2013-2718, 2013.

${ }^{12}$ Reibert, M.S. and Saric, W.S., "Review of Swept-Wing Transition," AIAA Paper 97-1816, 1997.

${ }^{13} \mathrm{Li}$, F. and Choudhari, M. M., "Stationary Crossflow Breakdown due to Mixed Mode Spectra of Secondary Instabilities," Submitted to AVIATION-2016 Conference, Washington, D.C., 2016.

${ }^{14}$ Belisle, M., Neale, T., Reed, H., and Saric, W. "Design of a Swept-Wing Laminar Flow Control Flight Experiment for Transonic Aircraft," AIAA paper 2010-4381, 2010

${ }^{15}$ Wie, Y.-S., "BLSTA - A Boundary Layer Code for Stability Analysis," NASA CR 4481, 1992.

${ }^{16}$ Paredes, P., Hanifi, A., Theofilis, V., and Henningson, D., "The nonlinear PSE-3D concept for transition prediction in Flows with a Single Slowly-Varying Spatial Direction," Procedia IUTAM, Vol. 14C, 2015, pp. 35-44.

${ }^{17}$ Chang, C.-L., "Langley Stability and Transition Analysis Code (LASTRAC) Version 1.2 User Manual," NASA/TM-2004213233, June, 2004

${ }^{18}$ Li, F., Choudhari, M., Duan, L., and Chang, C.-L. "Nonlinear Development and Secondary Instability of Traveling Cross Flow Vortices", Physics of Fluids, Vol. 26, 064104, 2014. 